\title{
Rendimentos do trabalho e de outras rendas e a queda da concentração de renda no Paraná
}

\author{
Solange de Cássia Inforzato de Souza* \\ Carlos Roberto Ferreira ${ }^{* *}$
}

Resumo: Este trabalho tem como objetivo estudar a influência do rendimento do trabalho e de outras rendas na queda da concentração de renda no estado do Paraná. A análise revela que houve (i) uma manutenção da estrutura das fontes de renda no estado, liderada pelo trabalho e pelas aposentadorias e pensões, (ii) aumento da participação dos rendimentos do trabalho e redução da importância das transferências condicionadas na renda domiciliar no quadriênio, contrariamente ao que se observa desde a década de 1990, (iii) e queda do índice de concentração de renda no estado.

Palavras-chave: Concentração de renda; Renda do trabalho; Renda das aposentadorias; Transferências de renda.

Classificação JEL: D33; I38; J26.

\footnotetext{
"Doutora em Educação: História, Política, Sociedade pela Pontifícia Universidade Católica de São Paulo (PUC-SP). Professora associada da Universidade Estadual de Londrina (UEL). Endereço eletrônico: solangecassia@uol.com.br.

** Doutor em Ciências Econômicas pela Universidade de São Paulo (USP). Professor associado da Universidade Estadual de Londrina (UEL). Endereço eletrônico: robert@uel.br. 


\section{Introdução}

As transformações da economia brasileira na última década, especialmente em sua segunda metade, refletiram sobre as condições de vida, de renda e de trabalho da sua população, materializadas no crescimento da renda das camadas mais pobres, na ascensão da classe média, na recuperação do mercado de trabalho e na redução da desigualdade da renda pessoal.

As experiências, no plano doméstico, do regime macroeconômico ancorado nas metas de inflação, responsabilidade fiscal e câmbio flutuante do pós-1999, do mecanismo robusto de transferência condicionada de renda pós2003, da política de valorização do salário mínimo e formalização do emprego, aliadas ao plano externo favorável pelo crescimento das exportações e choque positivo dos termos de troca até 2008 , em grande parte indicaram a relevância dos anos 2000 para a sociedade brasileira. As dificuldades enfrentadas pela crise financeira internacional de meados de 2008 abalaram os resultados econômicos e do mercado de trabalho em 2009, mas estes foram ainda melhores do que o temido no início da crise.

Apesar de ainda ostentar alto nível de concentração de renda, as medidas da desigualdade de renda no Brasil e, particularmente, no estado do Paraná, vêm apontando um declínio contínuo e acentuado desde 2001 (Soares, 2006; Hoffmann, 2005; Ferreira et al., 2006; Neri, 2006; Neri; Son, 2006; Barros et al., 2006a, 2006b; Ferreira; Souza, 2007).

A revisão da bibliografia, de modo geral, ora enaltece a importância de programas de transferência de renda, ora as mudanças no mercado de trabalho para a interpretação do comportamento da concentração de renda no Brasil. Neste sentido, a dimensão regional da desigualdade de renda é objeto relevante e particular de estudo, porque contempla as especificidades da unidade federativa.

Diante disso, este trabalho tem como objetivo estudar a influência do rendimento do trabalho e de outras rendas na queda da concentração de renda no estado do Paraná para os anos de 2006 e 2009. Mensura-se a participação desses rendimentos na renda domiciliar per capita e suas contribuições particulares para a melhoria da distribuição de renda, bem como a distribuição de cada um dos elementos formadores da renda domiciliar per capita em estratos de rendimento.

\section{Metodologia}

O presente estudo tem por base as informações coletadas na Pesquisa Nacional por Amostra de Domicílios (PNAD) em 2006 e 2009. Os componentes do rendimento domiciliar são os provenientes do trabalho principal e de outros trabalhos, de aposentadorias e pensões, dos aluguéis, doações, e outros rendimentos, apresentados para as pessoas de dez anos ou mais de idade.

Os procedimentos da pesquisa contemplaram a estratificação do rendimento domiciliar per capita em 11 estratos de renda e a decomposição do índice de Gini para o Brasil e Paraná em 2006 e 2009 proposto por Pyatt, Chen 
e Fei (1980).

No caso da decomposição, seja $z_{i}$ o rendimento da $i$-ésima pessoa. Neste trabalho, considera-se que o rendimento domiciliar per capita é o resultado da soma de $k$ parcelas:

$$
z_{i}=\sum_{h=1}^{k} z_{h i}
$$

Vamos admitir que os rendimentos $z_{i}$ estão ordenados de maneira que $z_{1} \leq z_{2} \leq \ldots \leq z_{n}$, sendo $n$ o tamanho da população. Então $i$ é a posição de ordem do rendimento $z_{i}$.

Pode-se verificar que o índice de Gini $(\mathrm{G})$ da distribuição de $z_{i}$ é:

$$
G=\frac{2}{n \mu} \operatorname{cov}\left(z_{i}, i\right)
$$

onde $\mu$ é a média dos $z_{i}$.

Pyatt, Chen e Fei (1980) definem a razão de concentração do componente $z_{h i}$ como:

$$
C_{h}=\frac{2}{n \mu_{h}} \operatorname{cov}\left(z_{h i}, i\right)
$$

onde $\mu_{h}$ é a média dos $z_{h i}$.

A participação do $h$-ésimo componente no rendimento total é:

$$
\phi_{h}=\frac{\mu_{h}}{\mu}
$$

Pode-se deduzir que:

$$
G=\sum_{h=1}^{k} \phi_{h} C_{h}
$$

Note-se, na expressão (5), que se todas as razões de concentração $C_{h}$ tivessem o mesmo valor, esse seria, também, o valor de $G$. Dessa forma, podese considerar que um componente $z_{h i}$ contribui para aumentar a desigualdade (medida pelo índice de Gini) quando $C_{h}>G$.

\section{Resultados da pesquisa}

A medida da participação das diferentes fontes de rendimento domiciliar per capita nas regiões selecionadas permite separar dois componentes importantes: um relacionado ao trabalho (trabalho principal e outros trabalhos) e outro ao rendimento das transferências de renda (aposentadorias e pensões, e outras rendas). Há o predomínio da renda do trabalho, no Paraná, que representou $78,4 \%$ e $79,5 \%$ do rendimento total em 2006 e 2009 , enquanto que as das transferências de renda somam $18,7 \%$ e $18,1 \%$ do total para os anos 
referidos, conforme a Tabela 1 .

Tabela 1 - Decomposição do valor médio (VM), em reais de abril de 2006 e 2009, e a participação percentual $\left[(\%) \phi_{k}\right]$ dos componentes do rendimento domiciliar per capita - Brasil e Paraná, 2006 e 2009

\begin{tabular}{|c|c|c|c|c|c|c|c|c|}
\hline \multirow{4}{*}{ Parcelas } & \multicolumn{8}{|c|}{ Valor médio e sua participação percentual } \\
\hline & \multicolumn{4}{|c|}{ Brasil } & \multicolumn{4}{|c|}{ Paraná } \\
\hline & \multicolumn{2}{|c|}{2006} & \multicolumn{2}{|c|}{2009} & \multicolumn{2}{|c|}{2006} & \multicolumn{2}{|c|}{2009} \\
\hline & VM & $\%$ & VM & $\%$ & VM & $\%$ & VM & $\%$ \\
\hline Trabalho principal & 356,67 & 72,8 & 465,44 & 73,0 & 415,56 & 75,0 & 559,39 & 76,1 \\
\hline Outros trabalhos & 15,52 & 3,2 & 20,31 & 3,2 & 18,61 & 3,4 & 25,01 & 3,4 \\
\hline $\begin{array}{l}\text { Aposentadorias e } \\
\text { pensões }\end{array}$ & 95,28 & 19,4 & 128,35 & 20,1 & 91,20 & 16,5 & 124,67 & 16,9 \\
\hline Aluguéis & 8,47 & 1,7 & 9,96 & 1,6 & 11,72 & 2,1 & 15,20 & 2,1 \\
\hline Doações & 3,47 & 0,7 & 2,81 & 0,4 & 4,44 & 0,8 & 2,53 & 3,4 \\
\hline Juros etc. & 10,63 & 2,2 & 10,79 & 1,7 & 12,45 & 2,2 & 8,67 & 1,2 \\
\hline Total & 490,03 & 100,0 & 637,67 & 100,0 & 553,98 & 100,0 & 735,54 & 100,0 \\
\hline
\end{tabular}

Fonte: Elaboração dos autores a partir da PNAD (2006 e 2009).

Ao operar a desagregação dos elementos que compõem o rendimento domiciliar per capita, pode-se perceber o que segue (ver Tabela 2). No estado do Paraná, em 2006 e 2009, os dois maiores componentes do rendimento não contribuem para a concentração de renda. Essa não é a realidade para o Brasil como um todo, evidenciando uma particularidade no estado, pois as aposentadorias continuam contribuindo para a concentração, ao contrário do Paraná.

Tabela 2 - Razão de concentração (C) na decomposição do índice de Gini do rendimento domiciliar per capita Brasil e Paraná, 2006 e 2009

\begin{tabular}{|c|c|c|c|c|}
\hline \multirow{3}{*}{$\begin{array}{l}\text { Componente do } \\
\text { rendimento }\end{array}$} & \multicolumn{4}{|c|}{ Razão de Concentração (C) } \\
\hline & \multicolumn{2}{|c|}{ Brasil } & \multicolumn{2}{|c|}{ Paraná } \\
\hline & 2006 & 2009 & 2006 & 2009 \\
\hline Trabalho principal & 0,555 & 0,534 & 0,505 & 0,487 \\
\hline Outros trabalhos & 0,760 & 0,773 & 0,755 & 0,787 \\
\hline Aposentadorias e pensões & 0,579 & 0,560 & 0,512 & 0,460 \\
\hline Aluguéis & 0,792 & 0,774 & 0,742 & 0,734 \\
\hline Doações & 0,445 & 0,391 & 0,467 & 0,382 \\
\hline Juros e outras transferências & 0,122 & $-0,105$ & 0,356 & 0,160 \\
\hline Gini total & 0,560 & 0,539 & 0,516 & 0,493 \\
\hline
\end{tabular}

Os rendimentos de outros trabalhos que não o principal representam a fonte de renda mais comprometida no país, pois que contribuem, juntamente com a renda dos aluguéis, para a concentração de renda.

$\mathrm{O}$ índice de Gini dos componentes do rendimento domiciliar per capita $\left[G\left(X_{k}\right)\right]$ pode ser visto na Tabela 3 . O rendimento do trabalho principal tem índice de Gini de 0,565 (2006) e 0,547 (2009) no estado, e a proporção de pessoas em domicílio com rendimento dessa fonte igual a zero é de apenas 8,6 (2006) e 9,2 (2009). No Brasil, o índice de Gini do trabalho é 0,616 (2006) e 0,598 (2009), e a proporção de pessoas referidas é de 9,9 (2006) e 10,6 (2009).

No caso das aposentadorias no estado, o índice de Gini é 0,854, e 68,4\% 
é a proporção das pessoas em domicílios cujo rendimento em tela não existe. Por outro lado, o índice de Gini da renda de outros trabalhos é de 0,974, com 92,7\% das pessoas em domicílios com esse rendimento igual a zero. Esses dados são interessantes também para o Brasil.

Tabela 3 - Índice de Gini dos componentes do rendimento domiciliar per capita $\left[G\left(X_{k}\right)\right]$ e proporção das pessoas em domicílios com rendimento per capita igual a zero - Brasil e Paraná, 2006 e 2009

\begin{tabular}{|c|c|c|c|c|c|c|c|c|}
\hline \multirow[t]{3}{*}{ Componente do rendimento } & \multicolumn{4}{|c|}{$G\left(X_{k}\right)$} & \multicolumn{4}{|c|}{$\begin{array}{l}\text { Proporção de pessoas } \\
\text { em domicílios com } \\
\text { rendimento per capita } \\
\text { igual a zero }(\%)\end{array}$} \\
\hline & \multicolumn{2}{|c|}{ Brasil } & \multicolumn{2}{|c|}{ Paraná } & \multicolumn{2}{|c|}{ Brasil } & \multicolumn{2}{|c|}{ Paraná } \\
\hline & 2006 & 2009 & 2006 & 2009 & 2006 & 2009 & 2006 & 2009 \\
\hline Trabalho principal & 0,616 & 0,598 & 0,565 & 0,547 & 9,9 & 10,6 & 8,6 & 9,2 \\
\hline Outros trabalhos & 0,976 & 0,977 & 0,974 & 0,974 & 92,7 & 93,1 & 92,7 & 93,2 \\
\hline Aposentadorias e pensões & 0,856 & 0,850 & 0,854 & 0,837 & 67,3 & 66,2 & 68,4 & 66,4 \\
\hline Aluguéis & 0,985 & 0,986 & 0,979 & 0,980 & 96,1 & 96,4 & 94,7 & 94,9 \\
\hline Doações & 0,991 & 0,993 & 0,992 & 0,995 & 97,1 & 97,8 & 97,8 & 98,7 \\
\hline Juros e outras transferências & 0,903 & 0,889 & 0,946 & 0,951 & 71,3 & 74,9 & 77,8 & 84,3 \\
\hline
\end{tabular}

Fonte: Elaboração dos autores a partir da PNAD (2006 e 2009)

Na análise da fonte de renda derivada do trabalho, é importante lembrar que se existe um quadro de ocupações cujo topo é estreito e sua base é larga, e se as ocupações do topo não absorvem toda a oferta de mão de obra, temse necessariamente um perfil concentrado para a distribuição dos rendimentos provenientes do trabalho, que é reforçado pela existência de um nível baixo de salários para a maior parte das pessoas pertencentes à base do mercado de trabalho.

Quanto menor o piso salarial do mercado de trabalho, maior tende a ser a desigualdade salarial possível de se verificar no sistema, uma vez que ela se estabelece em função da posição dos cargos e remunerações tão diferentes na estrutura produtiva.

Assim, é provável que esteja havendo uma retração na base ocupacional favorecendo a redistribuição de renda no Paraná. Os dados indicam que a política de valorização do salário mínimo federal, a política estadual de salário mínimo a partir de 2006 e a formalização do emprego podem ter influenciado o patamar menor de concentração de todas as rendas no Paraná, visivelmente menor do que a do Brasil.

A isso se acrescenta que não há contribuição da renda das aposentadorias e pensões nem das transferências condicionadas para a concentração da renda no estado, implicando em melhorias na distribuição desses rendimentos, conquanto as políticas sociais seguem em ampliação.

As Tabelas 4, 5 e 6 mostram a estratificação do rendimento domiciliar per capita. A Tabela 4 indica que a maior concentração percentual de domicílios na população do Brasil está no estrato de rendimento domiciliar per capita de mais de 0,5 a $1 \mathrm{SM}$, enquanto que a maior concentração percentual de pessoas na população está no estrato de mais de 0 a 0,5 SM. Ambos estão concentrados nos 
estratos inferiores da distribuição, ou seja, nos estratos onde estão localizados os relativamente pobres.

Tabela 4 - Distribuição dos domicílios e das pessoas em estratos de rendimento domiciliar per capita - Brasil, 2006 e 2009

\begin{tabular}{|c|c|c|c|c|c|c|c|c|c|c|}
\hline \multirow{3}{*}{$\begin{array}{c}\text { Estrato de } \\
\text { rendimento } \\
\text { domiciliar per } \\
\text { capita, em SM }\end{array}$} & \multicolumn{4}{|c|}{ Domicílios } & \multicolumn{4}{|c|}{ Pessoas } & \multirow{2}{*}{\multicolumn{2}{|c|}{$\begin{array}{c}\% \text { do } \\
\text { rendimento } \\
\text { total }\end{array}$}} \\
\hline & \multicolumn{2}{|c|}{2006} & \multicolumn{2}{|c|}{2009} & \multicolumn{2}{|c|}{2006} & \multicolumn{2}{|c|}{2009} & & \\
\hline & $\mathrm{N}^{\circ}\left(\mathbf{1 0}^{3}\right)$ & $\%$ & $\mathrm{~N}^{\circ}\left(10^{3}\right)$ & $\%$ & $\mathrm{~N}^{\circ}\left(10^{3}\right)$ & $\%$ & $N^{\circ}\left(10^{3}\right)$ & $\%$ & 2006 & 2009 \\
\hline 0 & 492,0 & 0,92 & 762,7 & 1,34 & 1197,5 & 0,66 & 1706,4 & 0,92 & 0 & 0 \\
\hline Mais de 0 a 0,5 & 13417,4 & 25,10 & 13194,9 & 23,24 & 59274,3 & 32,51 & 55686,9 & 30,13 & 7,0 & 6,6 \\
\hline Mais de 0,5 a 1 & 15188,3 & 28,41 & 16366,6 & 28,83 & 52071,2 & 28,56 & 54254,8 & 29,36 & 15,2 & 16,0 \\
\hline 1 a 1,5 & 8221,3 & 15,38 & 9139,1 & 16 & 256 & 14,07 & 27802,5 & 15,04 & 12,4 & 13,5 \\
\hline 1,5 a 2 & 4810,0 & 9,00 & 5611,9 & 9,89 & 13644,8 & 7,48 & 15299,4 & 8,28 & 9,3 & 10,5 \\
\hline e 2 a 2,5 & 2740,8 & 5,13 & 3029,9 & 5,34 & 8024,3 & 4,40 & 8345,9 & 4,52 & 7,0 & 7,4 \\
\hline 2,5 a 3 & 1969,2 & 3,68 & 1945,3 & 3,43 & & 2,96 & & 2,83 & 5,8 & 5,7 \\
\hline 3 a 4 & 2091,7 & 3,91 & 2326,2 & 4,10 & 5847,6 & 3,21 & 6150,8 & 3,33 & 7,9 & 9,0 \\
\hline Mais de 4 a 8 & 3032,5 & 5,67 & 3029,0 & 5,34 & 7869,9 & 4,32 & 7436,1 & 4,02 & 17,0 & 16,1 \\
\hline 8 a 15 & 1073,8 & 2,01 & 989,5 & 1,74 & 2519,5 & 1,38 & 2226,6 & 1,20 & 10,5 & 9,3 \\
\hline Mais de 15 & 416,9 & 0,78 & 371,5 & 0,65 & 839,9 & 0,46 & 678,5 & 0,37 & 7,9 & 6,5 \\
\hline Total & 53454,5 & 00,00 & $9 \pi c 7$ & 00,00 & 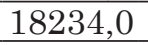 & מסת & 1.8488 & 00,00 & 00,0 & 100,0 \\
\hline
\end{tabular}

Fonte: Elaboração dos autores a partir dos dados da PNAD (2006 e 2009).

Para o Brasil, se considerarmos os estratos de rendimento domiciliar per capita de 0 a 1,5 SM, os dados mostram que 69,81\% (69,51\%) dos domicílios e $75,8 \%(75,45 \%)$ das pessoas que estão nessa faixa de renda têm rendimento per capita médio de até $\mathrm{R} \$ 432,61(567,84)$, representando $34,6 \%(36,1 \%)$ do rendimento total. No estrato de mais de 4 a 15 SM, tem-se 7,68\% (7,08\%) dos domicílios e $5,7 \%(5,22 \%)$ das pessoas que detêm $27,5 \%(25,4 \%)$ do rendimento total, com rendimento per capita médio de até $\mathrm{R} \$ 3.705,24(\mathrm{R} \$ 4.858,70)$. Os números entre parênteses referem-se ao ano de 2009.

No Paraná, para as mesmas faixas de renda, os valores são aproximados. No primeiro intervalo (0 a 1,5 SM) estão 65,42\% (63,31\%) dos domicílios e $70,54 \%(68,78 \%)$ das pessoas que recebem $32,8 \%(33,0 \%)$ do rendimento total do estado. Na segunda faixa (mais de 4 à $15 \mathrm{SM}$ ), onde situam-se 7,92\% (8,34\%) dos domicílios e $6,25 \%(6,69 \%)$ das pessoas, estão $25,6 \%(27,2 \%)$ dos rendimentos totais (ver Tabela 5).

A análise da concentração de renda desagregada pelos componentes do rendimento domiciliar per capita é observada na Tabela 6 . Vê-se que 33,73\% $(33,26 \%)$ dos rendimentos do trabalho principal no Paraná estão nos estratos inferiores (mais de 0 a 1,5 SM), enquanto que 24,5\% (26,9\%) deles estão nos estratos de mais de 4 a 15 SM. Também 28,85\% (24,87\%) dos rendimentos das aposentadorias e pensões estão nessa última faixa. Os dados revelam que a composição da renda sofreu ligeira modificação nesses dois anos: há pequena redução da participação dos rendimentos do trabalho principal nos estratos inferiores e aumento nos estratos superiores; e redução da participação das rendas das aposentadorias nos estratos superiores. A alta concentração de renda dos outros trabalhos é evidenciada na faixa de mais de 4 à $15 \mathrm{SM}$.

O recorte para a faixa dos rendimentos acima de 15 SM mostra que 
estão nessa faixa $7,72 \%$ e $4,07 \%$ dos rendimentos do trabalho principal e $3,95 \%$ e 3,97\% das aposentadorias e pensões, ao passo que também estão 20,67\% e $12,12 \%$ dos rendimentos de outros trabalhos, respectivamente para 2006 e 2009.

Tabela 5 - Distribuição dos domicílios e das pessoas em estratos de rendimento domiciliar per capita - Paraná, 2006 e 2009

\begin{tabular}{|c|c|c|c|c|c|c|c|c|c|c|}
\hline \multirow{3}{*}{$\begin{array}{c}\text { Estrato de } \\
\text { rendimento } \\
\text { domiciliar } \\
\text { per capita, em } \\
\text { SM } \\
\end{array}$} & \multicolumn{4}{|c|}{ Domicílios } & \multicolumn{4}{|c|}{ Pessoas } & \multirow{2}{*}{\multicolumn{2}{|c|}{\begin{tabular}{|c|}
$\%$ do \\
rendimento \\
total
\end{tabular}}} \\
\hline & \multicolumn{2}{|c|}{2006} & \multicolumn{2}{|c|}{2009} & \multicolumn{2}{|c|}{2006} & \multicolumn{2}{|c|}{2009} & & \\
\hline & $\mathrm{N}^{\circ}\left(10^{3}\right)$ & $\%$ & $\mathrm{~N}^{\circ}\left(10^{3}\right)$ & $\%$ & $\mathrm{~N}^{\circ}\left(10^{3}\right)$ & $\%$ & $\mathrm{~N}^{\circ}\left(10^{3}\right)$ & $\%$ & 2006 & 2009 \\
\hline 0 & 17,3 & 0,55 & 43,6 & 1,29 & 32,8 & 0,32 & 95,5 & 0,91 & 0 & 0 \\
\hline Mais de 0 a 0,5 & 557,4 & 17,67 & 483,9 & 14,26 & 2316,2 & 22,49 & 1955,2 & 18,58 & 4,6 & 3,9 \\
\hline Mais de 0,5 a 1 & 915,1 & 29,01 & 968,1 & 28,52 & 3079,1 & 29,89 & 3179,8 & 30,21 & 14,3 & 14,3 \\
\hline Mais de 1 a 1,5 & 573,7 & 18,19 & 652,9 & 19,24 & 1837,5 & 17,84 & 2008,1 & 19,08 & 13,9 & 14,8 \\
\hline Mais de 1,5 a 2 & 340,5 & 10,80 & 402,6 & 11,86 & 984,4 & 9,56 & 1099,0 & 10,44 & 10,6 & 12,0 \\
\hline Mais de 2 a 2,5 & 194,7 & 6,18 & 232,1 & 6,84 & 577,1 & 5,60 & 639,8 & 6,08 & 8,0 & 8,6 \\
\hline Mais de 2,5 a 3 & 127,3 & 4,04 & 136,4 & 4,02 & 353,8 & 3,43 & 368,2 & 3,50 & 6,0 & 6,0 \\
\hline Mais de 3 a 4 & 152,6 & 4,84 & 172,5 & 5,08 & 423,5 & 4,11 & 438,8 & 4,17 & 9,0 & 9,0 \\
\hline Mais de 4 a 8 & 189,6 & 6,01 & 224,4 & 6,61 & 512,6 & 4,98 & 571,9 & 5,43 & 17,2 & 19,0 \\
\hline Mais de 8 a 15 & 60,1 & 1,91 & 58,8 & 1,73 & 131,1 & 1,27 & 133,0 & 1,26 & 8,4 & 8,2 \\
\hline Mais de 15 & 25,2 & 0,80 & 18,5 & 0,55 & 52,1 & 0,51 & 35,0 & 0,33 & 8,0 & 4,8 \\
\hline Total & & & & & & & & 000 & 000 & 1000 \\
\hline
\end{tabular}

Fonte: Elaboração dos autores a partir dos dados da PNAD (2006 e 2009).

Tabela 6 - Distribuição de componente do rendimento domiciliar em estratos de rendimento domiciliar per capita - Brasil e Paraná, 2006 e 2009

\begin{tabular}{|c|c|c|c|c|c|c|c|c|c|c|c|c|c|c|}
\hline \multirow{3}{*}{$\begin{array}{c}\text { Estrato de } \\
\text { rendimento } \\
\text { domiciliar per } \\
\text { capita em SM }\end{array}$} & \multicolumn{14}{|c|}{ Rendimentos (\%) } \\
\hline & \multicolumn{2}{|c|}{$\begin{array}{l}\text { Todos os } \\
\text { trabalhos }\end{array}$} & \multicolumn{2}{|c|}{$\begin{array}{l}\text { Trabalho } \\
\text { principal }\end{array}$} & \multicolumn{2}{|c|}{$\underset{\text { trabalhos }}{\text { Outros }}$} & \multicolumn{2}{|c|}{$\begin{array}{l}\text { Aposenta- } \\
\text { dorias e } \\
\text { pensões }\end{array}$} & \multicolumn{2}{|c|}{$\begin{array}{c}\text { Aposenta- } \\
\text { dorias }\end{array}$} & \multicolumn{2}{|c|}{ Pensões } & \multicolumn{2}{|c|}{$\begin{array}{c}\text { Aluguéis, } \\
\text { doaçóes, } \\
\text { outras } \\
\text { rendas }\end{array}$} \\
\hline & 2006 & 2009 & 2006 & 2009 & 2006 & 2009 & 2006 & 2009 & 2006 & 2009 & 2006 & 2009 & 2006 & 2009 \\
\hline 0 & 0 & 0 & 0 & 0 & 0 & 0 & 0 & 0 & 0 & 0 & 0 & 0 & 0 & 0 \\
\hline Mais de 0 a 0,5 & 4,63 & 3,75 & 4,79 & 3,90 & 1,10 & 0,35 & 3,91 & 3,64 & 3,06 & 2,94 & 5,42 & 5,00 & 7,45 & 20,38 \\
\hline 0,5 a 1 & 13,83 & 13,56 & 14,25 & 14,01 & 4,40 & 3,57 & 16,58 & 18,33 & 15,42 & 18,02 & 20,84 & 19,54 & 14,92 & 25,83 \\
\hline Mais de 1 a 1,5 & 14,42 & 14,98 & 14,69 & 15,35 & 8,34 & 6,77 & 13,38 & 15,01 & 12,90 & 14,87 & 14,99 & 16,86 & 8,11 & 8,76 \\
\hline Mais de 1,5 a 2 & 10,59 & 11,30 & 10,80 & 11,53 & 5,80 & 6,00 & 11,69 & 12,37 & 11,72 & 12,12 & 12,44 & 14,55 & 6,55 & 4,96 \\
\hline Mais de 2 a 2,5 & 8,22 & 8,68 & 8,29 & 8,76 & 6,64 & 6,77 & 7,40 & 8,32 & 7,88 & 8,87 & 6,72 & 7,74 & 5,86 & 2,79 \\
\hline Mais de 2,5 a 3 & 6,20 & 6,23 & 6,22 & 6,32 & 5,77 & 4,16 & 5,79 & 5,31 & 5,42 & 4,61 & 6,83 & 7,25 & 3,59 & 1,90 \\
\hline Mais de 3 a 4 & 9,00 & 9,27 & 8,73 & 9,16 & 15,04 & 11,87 & 8,45 & 8,17 & 8,13 & 7,84 & 8,64 & 10,18 & 10,27 & 2,63 \\
\hline Mais de 4 a 8 & 17,02 & 19,09 & 16,75 & 18,82 & 23,07 & 24,92 & 17,95 & 18,38 & 19,70 & 19,38 & 13,10 & 12,96 & 17,02 & 7,19 \\
\hline Mais de 8 a 15 & 7,82 & 8,74 & 7,76 & 8,08 & 9,18 & 23,48 & 10,90 & 6,49 & 11,43 & 7,91 & 8,24 & 2,48 & 9,30 & 4,74 \\
\hline Mais de 15 & 8,27 & 4,42 & 7,72 & 4,07 & 20,67 & 12,12 & 3,95 & 3,97 & 4,34 & 3,46 & 2,80 & 3,44 & 16,93 & 20,82 \\
\hline Total & 100,0 & 100,0 & 100,0 & 100,0 & 100,0 & 100,0 & 100,0 & 100,0 & 100,0 & 100,0 & 100,0 & 100,0 & 100,0 & 100,0 \\
\hline
\end{tabular}

No estado do Paraná, duas são as considerações a se fazer. A primeira é a de que nos estratos inferiores da renda se encontram a maior parte dos domicílios e das pessoas, e aproximadamente $32 \%$ dos rendimentos são derivados do trabalho principal e $34 \%$ das aposentadorias e pensões. $\mathrm{Na}$ faixa mais alta dos estratos superiores (mais de 15SM) nitidamente o Paraná mostra que seus domicílios e pessoas estão distribuídos como no país, conforme mostra a literatura, mas o componente da renda aposentadorias e pensões para essa faixa é significativamente menor no estado. 
O mercado formalizado, os maiores vínculos previdenciários para os assalariados, a redução da taxa de desemprego, o aumento do rendimento das ocupações e a política de transferência de renda intensificada, sinalizam que os benefícios em termos de redução de desigualdades são intensos no estado do Paraná.

\section{Considerações finais}

Os dados confirmam a manutenção da estrutura das fontes de renda no estado, liderada pelo trabalho e pelas aposentadorias e pensões. No entanto, a queda da participação do trabalho desde o início dos anos 1990, apontada pelos estudiosos, é verificada, mas ocorre uma inversão dessa tendência quando se compara os anos de 2006 e 2009. O rendimento das aposentadorias e pensões, cuja participação fora incrementada naquela década, segue seu curso no período 2006-2009, e as rendas advindas das transferências condicionadas perdem espaço na renda domiciliar per capita no estado.

Adecomposição doíndice de Ginirevelou queda acentuada da concentração de renda no estado e confirmou que os rendimentos do trabalho principal e das transferências de renda (aposentadorias e pensões e outras transferências) não colaboram para essa concentração. Essa não é a realidade para o Brasil como um todo, captada pela literatura, evidenciando uma particularidade importante no estado.

A última consideração a se fazer é a de que, no Paraná, a maior parte dos domicílios e das pessoas se encontra nos estratos inferiores da renda, cujas rendas são derivadas do trabalho principal (em média 32\%) e das aposentadorias e pensões (em média 34\%). Na faixa mais alta dos estratos superiores (mais de $15 \mathrm{SM}$ ), nitidamente o Paraná mostra que seus domicílios e pessoas estão distribuídos como no país, mas o componente da renda das aposentadorias e pensões para essa faixa é menor no estado.

\section{Referências}

Barros, R.; Carvalho, M.; Franco, S.; Mendonça, R. (2006a). Consequências e causas imediatas da queda recente da desigualdade de renda brasileira. Rio de Janeiro: IPEA.

Barros, R.; Carvalho, M.; Franco, S.; Mendonça, R. (2006b). Uma análise das principais causas da queda recente na desigualdade de renda brasileira. Rio de Janeiro: IPEA.

Ferreira, C. R.; Souza, S. C. I. (2007). "O comportamento da desigualdade de renda no Paraná de 2001 a 2005”. Revista Paranaense de Desenvolvimento 112: 31-45.

Ferreira, F.; Leite, P.; Litchfield, J.; Ulyssea, G. (2006). "Ascensão e queda da desigualdade de renda no Brasil”. Econômica 8(1): 147-169.

Hoffmann, R. (2005). “As transferências não são a causa principal da redução na 
desigualdade”. Econômica 7(2).

IBGE - Instituto Brasileiro de Geografia e Estatística. (2006, 2009). Pesquisa Nacional por Amostra de Domicílios (CD). Rio de Janeiro.

Neri, M. (2006). "Rendas, bolsas e queda da desigualdade". Conjuntura econômica, julho.

Neri, M.; Kakwani, N.; Son, H. (2006). "Ingredientes trabalhistas na culinária da estagnação”. Conjuntura econômica, agosto.

Pyatt, G.; Chen, C.; Fei, J. (1980). "The distribution of income by factor components". The Quarterly Journal of Economics 95(3): 451-473.

Soares, S. S. D. (2006). Distribuição de renda no Brasil de 1976 a 2004 com ênfase no período entre 2001 e 2004. Rio de Janeiro: IPEA. 
